\title{
Impact of sample thickness on atomic resolution tomography by HAADF-STEM
}

\author{
Dmitri O. Klenov and Susanne Stemmer
}

Materials Department, University of California, Santa Barbara, CA 93106-5050

High-angle annular dark-field in scanning transmission electron microscopy is attractive for atomic resolution imaging of materials imperfections due to the absence of contrast reversals. The sensitivity of atomic column image intensities to the atomic number (Z-contrast) makes it an ideal tool for structure analysis. Recently, two methods have been proposed for atomic resolution tomography: (i) discrete tomography using atomically resolved images recorded along different directions and (ii) through-focus tomography, i.e. reconstruction from a through focus series [1, 2]. The latter technique is particularly attractive in conjunction with the development of $\mathrm{C}_{\mathrm{s}}$ correction where the focal depth is reduced by an order of magnitude [3]. Channeling and multiple scattering, however, may place limitation on both methods.

Here we present an experimental study of the contrast in atomically resolved HAADF-STEM images. In particular, we established the region of the TEM foil, which produces atomic column resolved contrast in non- $\mathrm{C}_{\mathrm{s}}$ corrected HAADF-STEM images. Two heterostructures were investigated: epitaxial $\mathrm{PbTiO}_{3}$ films on $\mathrm{SrTiO}_{3}$ and epitaxial InGaAs films on InP. The two heterostructures combined materials with the same crystal structure but different atomic numbers. Figure 1 shows HAADF-STEM images of $\mathrm{SrTiO}_{3}$ and $\mathrm{PbTiO}_{3}$ from regions with different thickness. Figure 2 shows the intensity of the atomic columns after subtraction of the background as a function of thickness. The intensity of the atomic columns increased until about $60-100 \mathrm{~nm}$ and then saturated. The saturation was a result of dechanneling. Consequently, atomically resolved HAADFSTEM image contrast originates only from the top surface of the TEM foil. Atom column intensities as a function of focus are shown in Figs. 3 and 4. An optimum focus exists for which the HAADFSTEM images show the highest contrast and column intensities. Away from this focus the intensities decrease and atom columns become indistinguishable from the noise (Fig. 3). The width of the peak in Fig. 4 is proportional to the channeling depth and was similar for TEM foils that were up to $340 \mathrm{~nm}$ thick. Most of the electrons of an underfocused beam are being channeled immediately after the probe enters the sample surface rather than being focused inside the TEM foil. A Cs corrected TEM might provide the images with much better signal-to-noise ratio from the thicker region if there are more electrons able to penetrate without strong channeling. The results also impact tomography by the first method, where the projected thickness of the sample increases by orders of magnitude during sample tilting. In particular, to fulfill the projection requirement of tomography, namely that image intensities are a monotonous function of thickness, the total sample thickness projected along the beam direction may not exceed $60-100 \mathrm{~nm}$ [4].

\section{References}

[1] C. Kisielowski, B. Kabius, C. Kiely, R. Phaneuf, and F. Ross, "Materials research in an Aberration-Free Environment (http://ncem.lbl.gov/team3.htm)," (2005).

[2] M. Varela, A. R. Lupini, K. van Benthem, A. Y. Borisevich, M. F. Chisholm, N. Shibata, E. Abe, and S. J. Pennycook, Ann. Rev. Mater. Res. 35, (2005) 539.

[3] K. van Benthem et al., Appl. Phys. Lett. 87 (2005) 034104. 
[4] This research was supported by the DOE Office of Basic Energy Sciences (grant \#DE-FG0302ER45994). The authors thank Prof. Paul McIntyre for the $\mathrm{SrTiO}_{3} / \mathrm{PbTiO}_{3}$ sample.

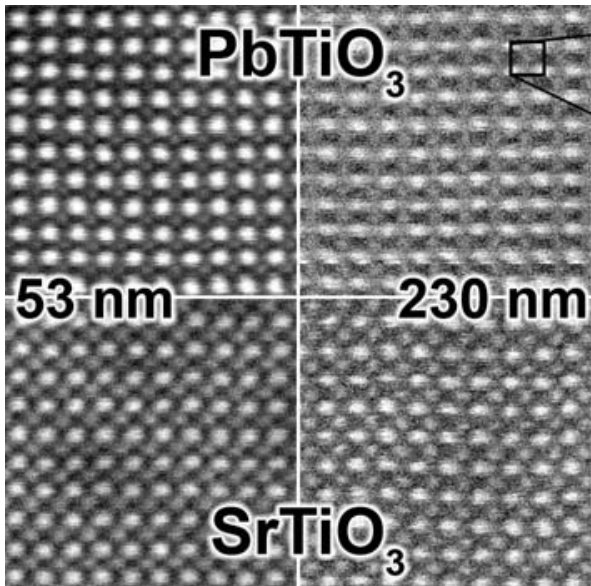

FIG. 1. HAADF-STEM images recorded from different foil thicknesses.
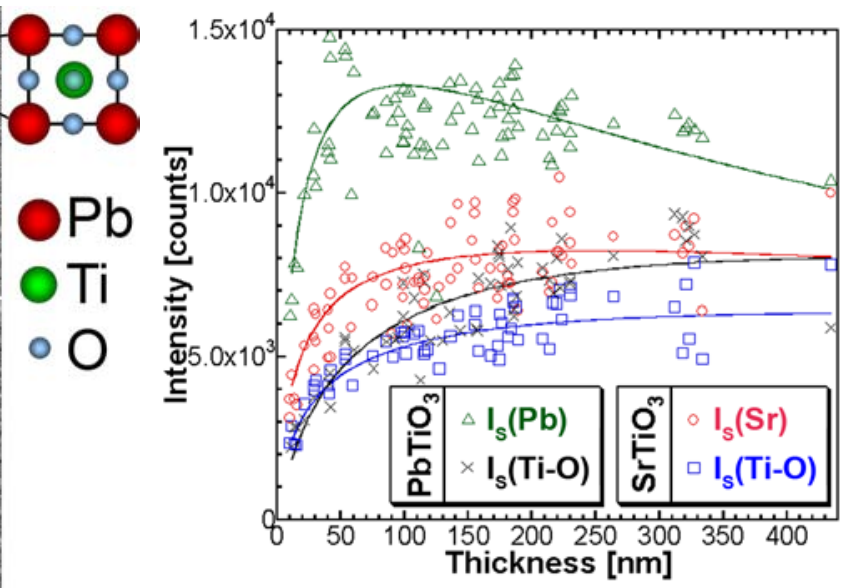

FIG. 2. Intensities of the atomic columns in $\mathrm{SrTiO}_{3}$ and $\mathrm{PbTiO}_{3}$ plotted as a function of the TEM foil thickness after subtraction of the background.
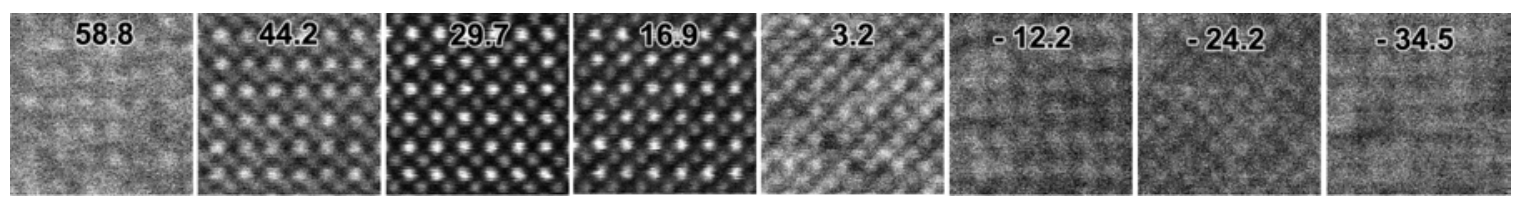

FIG. 3. Selected HAADF-STEM images of $\mathrm{SrTiO}_{3}$ from a focus series
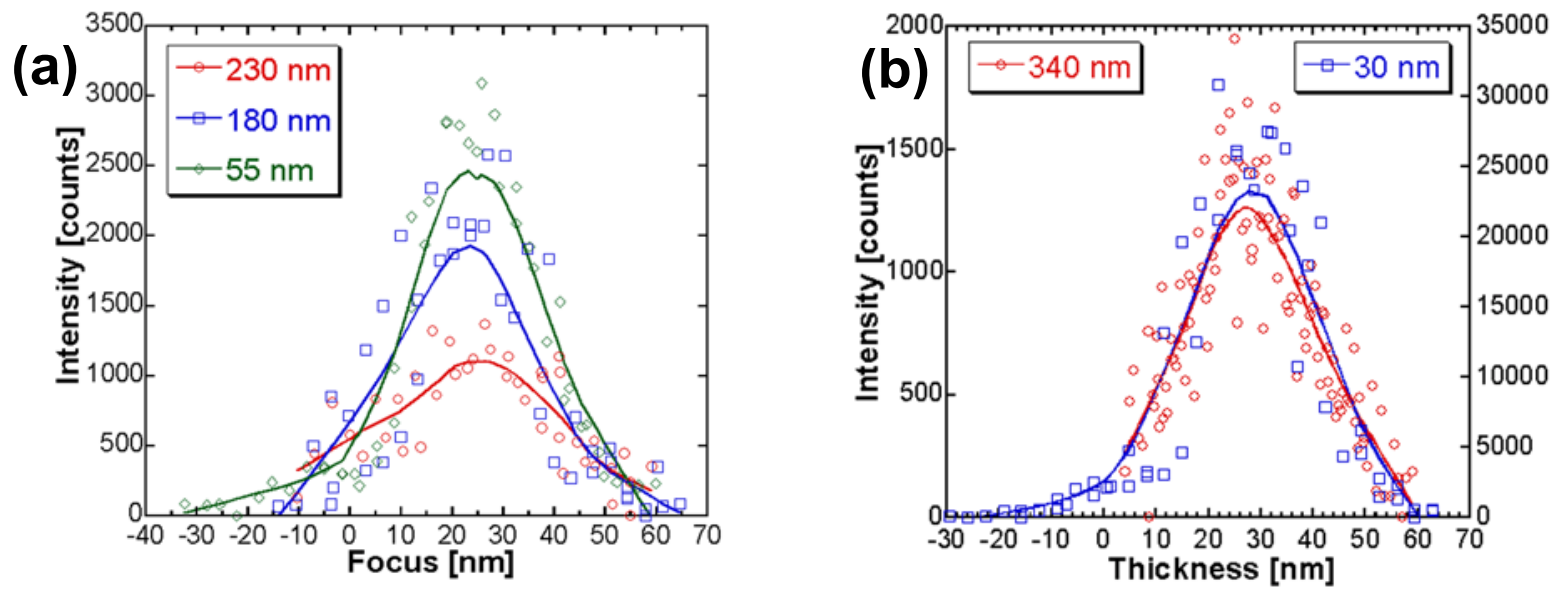

FIG. 4. Intensity of the atomic columns in (a) InP and (b) $\mathrm{SrTiO}_{3}$ plotted as a function of the focus for the different TEM foil thicknesses. Lines represent weighted fit and are shown as a guide for the eye. 